\title{
Critical current measurement with spatial periodic bending imposed by electromagnetic force on a standard test barrel with slots
}

\author{
A. Nijhuis, W. A. J. Wessel, Y. Ilyin, A. den Ouden, and H. H. J. ten Kate \\ Low Temperature Division, Faculty of Science and Technology, University of Twente, P.O. Box 217, \\ 7500 AE Enschede, The Netherlands
}

(Received 22 December 2005; accepted 19 March 2006; published online 3 May 2006)

\begin{abstract}
We have developed and validated a straightforward and fast method to investigate the response of technological superconducting strain sensitive wires (e.g., $\mathrm{Nb}_{3} \mathrm{Sn}$ ) to a spatial periodic bending strain. In the present concept of cabled superconductors for application in nuclear fusion reactors the wires are twisted and cabled in several stages. When subjected to transverse electromagnetic forces after charging the magnet, the individual strands are subjected to spatial periodic bending with wavelengths in the order of 5-10 $\mathrm{mm}$. Several apparatuses are presently under development to study the effect of bending on the transport properties, i.e., the voltage-current transition in terms of critical current $\left(I_{c}\right)$ and $n$ value. We propose a supplementary simple method to investigate the influence of bending strain by using a spatial periodic wire support on a broadly used standard $I_{c}$ measurement barrel in combination with a Lorentz force. The bending force $(B I)$ is varied by changing the applied field $B$. The peak bending strain in the $\mathrm{Nb}_{3} \mathrm{Sn}$ filaments is determined by the amplitude of the bending deflection, which is deduced from the mechanical axial tensile stress-strain properties of the wire. Three different spatial periodic wavelengths are applied and the results are in good agreement with the predictions. In addition we found a good agreement with results obtained by a more advanced experiment, named TARSIS, which is described briefly. The "barrel-with-slots" method can be applied easily and straightforward with minor effort and cost in laboratories having a standard $I_{c}$ measurement facility for superconducting wire. (C) 2006 American Institute of Physics. [DOI: 10.1063/1.2195118]
\end{abstract}

\section{INTRODUCTION}

It is known for already several decades that strain affects the performance of $\mathrm{Nb}_{3} \mathrm{Sn}$ based superconductors. ${ }^{1}$ Knowledge of the strain sensitivity of several types of A15 and HTc class superconductors is of major importance because it changes the transport properties under the influence of stress caused by, e.g., Lorentz loads in practical applications such as magnets. In particular for cable-in-conduit conductor (CICC) type of multistage cabled superconductors, as designed for the international thermonuclear experimental reactor (ITER), the wires are cabled in several stages and when subjected to electromagnetic forces, the individual strands are subjected to strain variations. The ITER model coil test results, for example, showed the conductor performance depends on the electromagnetic forces. ${ }^{2,3}$

Various approaches to characterize superconducting behavior versus strain have been employed throughout the last decades. For technological wire most attention has been paid to axial and transverse strain experiments as these represent the strain components relevant for magnet technology.

Influence of the axial strain can be studied with having a force applied on a free suspended sample, ${ }^{1,4}$ a sample mounted on a spiraled substrate, ${ }^{5,6}$ (so-called Walters spring), and a method based on a straight beam section that is part of a U-shaped bending spring ${ }^{7}$ or alternatively to an initially curved beam ${ }^{8}$ (so-called pacman). For applied transverse strain, we can distinguish between homogeneously distrib- uted loads on a wire, being, for example, sandwiched between flat surfaces, ${ }^{9,10}$ spatial periodically applied stress by using crossing strands, ${ }^{11,12}$ and finally bending strain. For bending strain we can differentiate between spatial periodical loading with a wavelike bending pattern 13,14 and bending along a bend surface or cylinder with constant radius. ${ }^{15-19}$ As bending strain in the $\mathrm{Nb}_{3} \mathrm{Sn}$ filaments is largely a distribution of axial strain over the wire cross section, knowledge of the $I_{c}$ versus axial strain dependency is required for analysis of the bending strain influence.

The interest for bending strain in $\mathrm{Nb}_{3} \mathrm{Sn}$ wires is highly stimulated after the analysis of the first model coil tests for ITER, built with large size CICC's, in 2000. ${ }^{2,3,20}$ Since that time, there seems to be a rather broad consensus on the effect of bending due to electromagnetic forces on the performance degradation of $\mathrm{Nb}_{3} \mathrm{Sn}$ wires in large multistage cabled CICC's. It is well established that a bending strain affects the critical current density of the filaments because of varying strain in axial direction of the twisted filaments. This initiates a decrease of the critical current, a broadening of the voltagecurrent transition (i.e., a drop in the $n$ value), or both. The bending strain response depends on the internal strand layout in terms of twist pitch and on the internal resistance, determining the degree of current transfer between the filaments.

The axial and transverse electromagnetic forces in combination with the thermal contraction in multistrand ITER conductors cause changes in the strand stress state and leads to elastoplastic strand deformations. The thermal loads arise 
due to the differential expansion between the $\mathrm{Nb}_{3} \mathrm{Sn}$ strand bundle and the steel or Incoloy conduit. The limited degree of flexibility for the strands in the cable together with the prebend wavy pattern from the cabling creates scope for not only axial precompression but possibly even cool down stresses acting like a bending strain.

The transverse forces lead to compressive pinching strain at the crossover contacts ${ }^{21}$ and bending strain along the strands. A hypothesis from Mitchell ${ }^{22}$ adopts bending as the main source for ITER conductor degradation and the predicted bending strains due to the transverse magnetic loads are up to $\pm 0.5 \%$. $^{3}$

In the framework of ITER research and development at our institute an apparatus named TARSIS has been developed to study the effect of various deformations such as homogeneous and spatial periodic transverse stress, and bending and axial (stress-) strain on the transport properties of superconducting wires. ${ }^{12,14}$ With the developed probes we are able to monitor, besides the voltage and current, precisely the actual force and deflection in terms of wire bending or deformation at three different locations on the wire itself. A detailed description of the setup and the results on bending of a powder-in-tube processed $\mathrm{Nb}_{3} \mathrm{Sn}$ wire are recently reported. ${ }^{23} \mathrm{~A}$ set of axial tensile stress-strain measurements on several strand types, necessary to analyze the results on bending, has just been published. ${ }^{24}$

Other recent experimental results in literature, in particular, on bending of $\mathrm{Nb}_{3} \mathrm{Sn}$ strands, show dispersion in results, basically depending on the applied experimental method. Therefore, a direct comparison of results is mostly inappropriate because some methods use prebending at room temperature, while others obtain precompression utilizing a material with a high coefficient of thermal expansion (CTE).

An approach that utilizes repeated bending at room temperature, as a method to improve the transport properties of $\mathrm{Nb}_{3} \mathrm{Sn}$ wires, causes an increase of the $I_{c}{ }^{25}$ (up to a factor of 2 at high fields) with a prebending strain up to $0.5 \%$. The bending strain is defined as the peak strain at the outer circumference of the wire's filamentary region. The observed increase in $I_{c}$ is attributed to a relaxation of the filament axial compressive strain due to an overall elongation of the wire, likely in combination with a reduction in radial prestress as well. Another experiment, ${ }^{16}$ investigating the impact of bend strain (bending just once and on a constant radius) of up to $0.3 \%$, applied at room temperature, revealed an $I_{c}$ improvement, exceeding 30\%. An experiment with applied spatial periodic bending at $4.2 \mathrm{~K}$, almost similar to the method applied with TARSIS except for a precise measurement of the bending amplitude, leads to only a decrease in $I_{c}$ with increasing bending deflection. A drawback of this method is that the exact zero strain condition is defined to coincide with the first observation in $I_{c}$ decrease. ${ }^{13}$ This approach may be argued since the initial bending of the wire goes with very low force and considering the results from other bending experiments, summarized above, we cannot exclude that the $I_{c}$ remains practically constant at low bending strain or may even show some increase. Adopting the initial change of the critical current as an indicator for the zero strain level may be misleading in the possible presence of strain relaxation

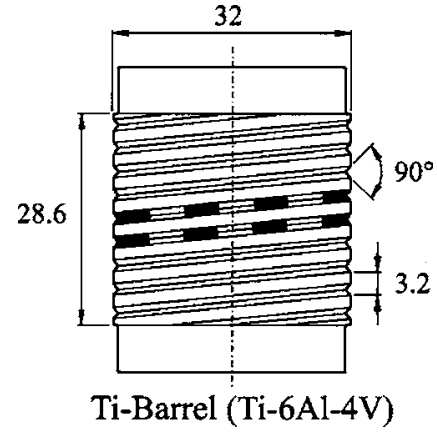

FIG. 1. Standard ITER sample holder with grooves to guide the wire. The black areas in the two center grooves represent the slots where the wire is not supported.

counterbalancing a decrease in $I_{c}$ due to bending. A precise measurement of the deflection is therefore crucial to resolve exactly the zero strain.

In summary, the variety in methods for wire bending experiments still leaves ample opportunity for different interpretations. For this reason we worked out an alternative route for exploring experimentally the effect of periodic bending on the $I_{c}$ of a $\mathrm{Nb}_{3} \mathrm{Sn}$ strand that can easily be used against low development cost by laboratories with an $I_{c}$ measurement facility. The method is indicated as "barrel with slots," from here on referred to as BWS. We give a description of the method and present the results obtained on a powder-intube processed $\mathrm{Nb}_{3} \mathrm{Sn}$ wire. The data determined with a background field of $12 \mathrm{~T}$ are compared with data taken from the same strand type on the TARSIS bending probe. ${ }^{23}$

\section{EXPERIMENTAL METHOD}

\section{A. Test configuration}

We use a well-known standard ITER barrel ${ }^{26}$ that is broadly applied for critical current measurements during the last decade. The strand is wound on the spiral groove of a molybdenum sulfide coated and preoxidized TiAlV cylinder, whose CTE matches closely that of $\mathrm{Nb}_{3} \mathrm{Sn}$. The wire is standard wound under tension with a counterweight of $0.8 \mathrm{~kg}$ to assure a constant sample pretension and defined preparation procedure. Slots are machined on recurring distances in the wall of the cylindrical sample holder at the two turns in the middle to allow for periodic bending when subjected to electromagnetic (EM) forces pointing towards the center of the barrel (see Fig. 1).

Basically the wire follows a periodic support of bridges with open spaces in between. The span of the slots is identical to the distance between them, determining the bending periodicity or wavelength (see Fig. 2). To restrict wire movement during the heat treatment, the wire ends are fixed by screws on the two copper rings attached to the TiAlV barrel. After the heat treatment the wire ends are soldered to the copper rings to which the current leads are connected (see Fig. 2).

The sample is shunted by a parallel superconducting wire (by soldering) at the transfer point from the barrel to the copper rings for connection to the current leads to provide better stability along this section. 

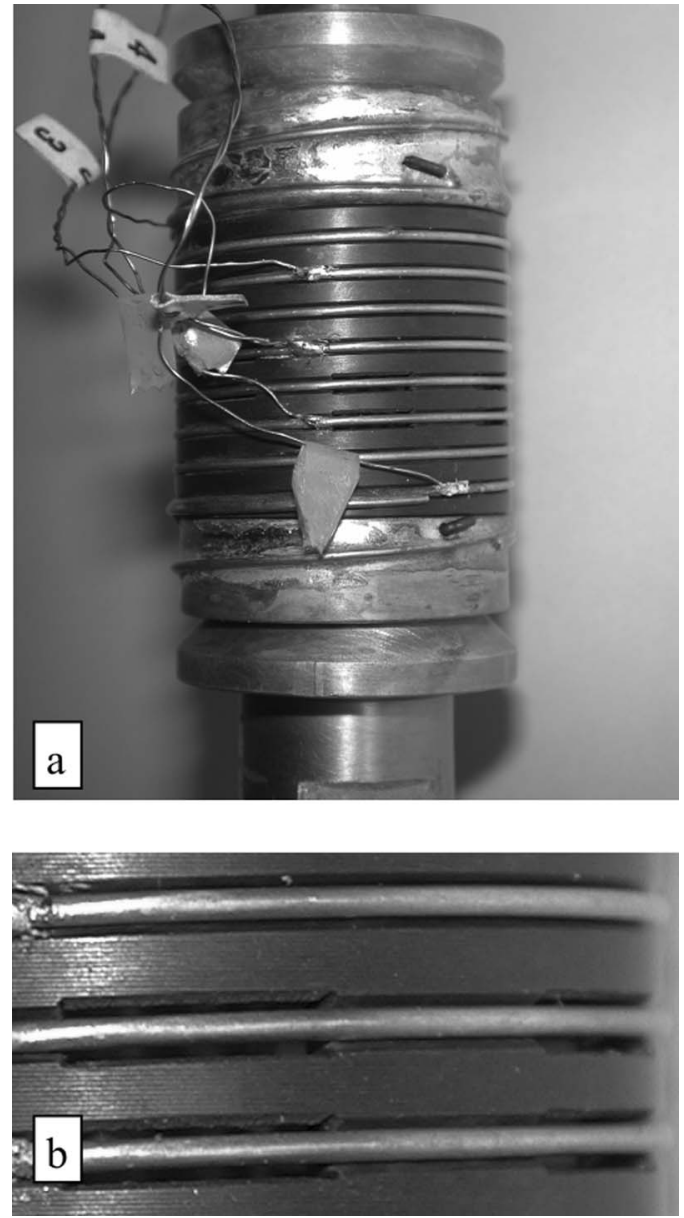

FIG. 2. Standard ITER sample holder with slots along two turns in the middle. The ends of the wire sample are soldered to the copper rings and the voltage taps are visible (a). Detailed view of the slots under the wire in the two grooves from the barrel with wire (b).

When the electromagnetic forces on the windings point inwards to the center of the barrel, the turns crossing the slots are subjected to bending. The other turns, supported along their entire length, reflect the properties of an unbent wire. The wire is not glued to the barrel and the depth of the groove assures a good guidance of the turns. Voltage taps are attached to both the sections subjected to spatial periodic bending and to the fully supported sections. The overall voltage of the sample including the section soldered to the current leads is measured as well to monitor the connection quality.

\section{B. Strand samples and test procedure}

One of the strands, tested in the TARSIS setup to investigate the impact of periodic bending, is a binary powder in tube (PIT) wire manufactured by Shape Metal Innovation (SMI). The same strand, ${ }^{23}$ with a diameter of $0.805 \mathrm{~mm}$ and 504 filaments, was used here for testing with the BWS method. Three barrels were prepared with slot wavelengths of 5.0, 7.2, and $9.1 \mathrm{~mm}$, being in the range of the bending wavelengths in the ITER model coil conductors. ${ }^{23}$ All samples are heat treated in one batch, no changes in the wire tension is applied after the heat treatment and during the soldering of the wire ends to the copper rings.
The test is performed with an extended field range starting at $15 \mathrm{~T}$ and subsequently decreasing with steps of $1 \mathrm{~T}$ to finally $7 \mathrm{~T}$. First the entire field range (down) with EM force inwards was explored and then repeated with the force pointing outwards (starting again at $15 \mathrm{~T}$ ).

\section{BENDING STRAIN EFFECT ON $I_{c}$}

\section{A. Low and high transverse resistivity regime}

The prediction of the effect of bending strain on the $I_{c}$ of a $\mathrm{Nb}_{3} \mathrm{Sn}$ strand is described by Ekin ${ }^{15}$ in terms of uniform bending (with constant radius) which creates a periodic strain variation along the twisted filaments. The model also describes quite well the effect of spatial periodic bending for a $\mathrm{Nb}_{3} \mathrm{Sn}$ strand with low transverse resistivity ${ }^{23}$ as the bending effect is not only affected by the applied bending strain but depends also on the interfilament electrical resistivity. The electrical resistance between the twisted filaments determines the current transfer length. The details are pointed out elsewhere ${ }^{15,23}$ so here we just give a brief summary.

When the wire is bent with a single radius in one direction, the twist of the filaments determines the periodicity in strain variation. In a cable, however, the pattern of crossing strands superimposes a cyclic varying strain pattern on top of the global cable twist. For the average spacing between the support points, about $6 \mathrm{~mm}$ are anticipated from a cable inspection on a CS1 type of ITER model coil $\operatorname{conductor}^{23}$ (the wavelengths in this periodic pattern may vary with the chosen twist pitches of the initial cabling stages). Current transfer between filaments therefore depends on both the resulting longitudinal strain periodicity and the interfilament resistance.

One (unlikely) extreme is that current transfer between the filaments is not allowed either by a very high matrix resistivity or a very short filament twist pitch. In this limiting case we may write ${ }^{15}$

$$
I_{c}=\frac{2 A}{\varepsilon_{b 0}^{2}} \int_{0}^{\varepsilon_{b 0}} J_{c}\left(B, T, \varepsilon_{t h}-\varepsilon\right) \varepsilon d \varepsilon \quad(\mathrm{A}),
$$

with $A$ the cross section area of the strand, $\varepsilon$ the strain in the filaments over a cross section of the strand, $\varepsilon_{b 0}$ the peak bending strain in the outer ring of filaments, $\varepsilon_{\mathrm{th}}$ the thermal precompression of the $\mathrm{Nb}_{3} \mathrm{Sn}$ filaments, and $J_{c}$ the critical current density. If on the other hand current transfer is allowed at a low voltage level, the overall $I_{c}$ of a strand is the sum of the filament currents at any section considering the local strain variation over the section. In this other limiting case, for (long pitch or) low matrix resistivity, which is likely more representative for most practical wires, we may write ${ }^{15}$

$$
I_{c}=\frac{2 A}{\pi \varepsilon_{b 0}^{2}} \int_{-\varepsilon_{b 0}}^{\varepsilon_{b 0}} J_{c}\left(B, T, \varepsilon_{t h}+\varepsilon\right) \sqrt{\varepsilon_{t h}^{2}-\varepsilon^{2}} d \varepsilon \quad \text { (A). }
$$

In order to solve relations (1) and (2) one needs to know the axial $I_{c}(\varepsilon)$ relation, which is obtained experimentally. ${ }^{8}$ The shape of the computed curves from relations (1) and (2) is primarily driven by the $I_{c}$ strain variation of the particular $\mathrm{Nb}_{3} \mathrm{Sn}$ strand. The state of the art $I_{c}(\varepsilon)$ scaling relations do not account for irreversible behavior in the $I_{c}(B, T, \varepsilon)$ data spectrum at high tensile loads and hence we use a polyno- 
mial fit to account for the additional large $I_{c}$ reduction at high tensile strain due to filament cracks. In Ref. 23 a polynomial fit to the experimental $I_{c}(\varepsilon)$ data is presented for the SMIPIT wire at $12 \mathrm{~T}$ and $4.2 \mathrm{~K}$. The data are measured with the pacman strain probe ${ }^{8}$ using an $I_{c}$ criterion of $10 \mu \mathrm{V} / \mathrm{m}$. Based on this polynomial $I_{c}(\varepsilon)$ fit, we calculate the bending effect extremes, assuming full or no interfilament current transfer for the strand $I_{c}$ range.

\section{B. Current transfer length and $\boldsymbol{n}$ value}

The current transfer length $L_{\mathrm{ct}}$ for low and high strand transverse resistances can be formulated for a certain voltage criterion as

$$
L_{\mathrm{ct}}=\left(\frac{0.1}{n}\right)^{0.5}\left(\frac{\rho_{\perp}}{\rho^{*}}\right)^{0.5} d,
$$

in which $n$ is the $n$ value characterizing the $V$ - $I$ transition, $\rho_{\perp}$ is the matrix resistivity, $\rho^{*}$ is the resistivity criterion used to define the $I_{c}$ (at $10 \mu \mathrm{V} / \mathrm{m}$ ), and $d$ is (throughout the article) the strand diameter. ${ }^{27}$ In this relation the resistivity and the $n$ value change during strand deformation due to yielding of the copper stabilizer but it is verified that the effect is only marginal in a bending experiment with a low number of cycles. $^{23}$

The longitudinal electric field in a strand $E_{z}$ is a nonlinear function of the current, of the temperature, and of the magnetic field. The $n$ values are normally determined from the $V-I$ curves in the electric field range of $10-100 \mu \mathrm{V} / \mathrm{m}$ with the commonly used power law $V-I$ representation. The $V-I$ characteristic, as the current increases, gradually travels from limited to full current transfer and the characteristic strongly depends on the bending strain and interfilament resistivity. Routinely we assume strands to have a constant $n$ value obeying a power law fit but for increasing periodic bending strain, the $V-I$ curve turns from (relatively) higher resistive to lower resistive regime along the $V$-I transition region (with increasing current) and does not actually follow anymore the power law. ${ }^{23}$ This illustrates the deficiency in application of the simple power law approach for the $n$ value in the interpretation of these bending experiments. Nevertheless, we will use the common formulation of the $n$ value, reflecting the steepness of the $V-I$ transition between 10 and $100 \mu \mathrm{V} / \mathrm{m}$, in order to avoid confusion in discussions with reference to the traditional definition.

The intrastrand transverse resistance is derived from the strand coupling-loss time constant. The constant $\tau$ of the coupling-loss per unit volume of strand is described as

$$
\tau=\frac{\mu_{0}}{2 \rho_{\perp}}\left(\frac{L_{p}}{2 \pi}\right)^{2} \quad(\mathrm{~s})
$$

where $L_{p}$ is the twist pitch, $\rho_{\perp}$ is the effective electrical transverse resistivity $(\Omega \mathrm{m})$, and $\mu_{0}$ is the permeability in vacuum. $^{28,29}$ The electromagnetic loss in superconductors consists of hysteresis and coupling loss. The coupling loss time constant is derived from the initial slope of the loss curve $\left[\left(\mathrm{J} / \mathrm{m}^{3}\right)\right.$, energy loss per cycle versus applied sweep rate of identical amplitude], assuming that the hysteresis loss per cycle at low excitation is independent from the sweep rate. This method of determining the transverse resistance
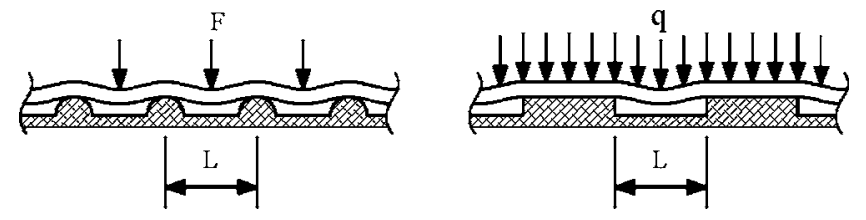

FIG. 3. Schematic view of point load (TARSIS bending probe) and distributed load (BWS method) with $L=L_{w}$.

between filaments from the inductive coupling between filaments is considered correct as also for bending, this particular interfilament coupling is relevant for redistribution. When the current transfer length is calculated with $\rho^{*}=1.7$ $\times 10^{-14} \Omega \mathrm{m}$ and an $n$ value of $50, L_{\mathrm{ct}}$ amounts to $4 \mathrm{~mm}$. With a filament twist pitch of $10 \mathrm{~mm}$ we expect the low resistivity scenario for this PIT strand.

\section{EVALUATION OF BENDING AMPLITUDE AND STRAIN}

The principle of the TARSIS bending probe with point loads and clamped ends is sketched in the left part of Fig. $3 .^{23}$ The point load $F$ represents the transferred magnetic point load from the upper crossing strand in a cable or as utilized by the periodic pattern with bulges in the TARSIS bending probe. The bending moment distribution is easily calculated from the theory of straight indeterminate beams that can be found in many textbooks.

The corresponding standard (elastic) equation for the deflection $(f)$ in the case of point loads $F$, with clamped ends and length $L$ (equal to the wavelength $L_{w}$ ) in Fig. 3, representing the TARSIS test state is given by

$$
f_{\max }=\frac{F L_{w}^{3}}{\alpha E I_{a}} \quad(\mathrm{~m}),
$$

in which the term $\alpha$ is 192 for point load, $E$ is the modulus of elasticity, and $I_{a}$ is the moment of inertia:

$$
I_{a}=\frac{\pi d^{4}}{64} \quad\left(\mathrm{~m}^{4}\right) .
$$

The peak strain $\varepsilon$ is now calculated with

$$
\varepsilon=\frac{M_{b}}{W_{b} E} \quad(-),
$$

in which $M_{b}$ represents the bending moment:

$$
M_{b}=\frac{F L_{w}}{\beta} \quad(\mathrm{N} \mathrm{m}),
$$

while $W_{b}$ is the section factor:

$$
W_{b}=\frac{\pi d^{3}}{32}, \quad\left(\mathrm{~m}^{3}\right) .
$$

The term $\beta$ in relation (8), giving the bending moment, depends on the position in the wire and is also given in general available textbooks. For the principle of the TARSIS bending probe with point load and clamped ends, the value for $\beta$ is 8 .

The principle of bending load from distributed electromagnetic force due to the strand currents is schematically represented in the right sketch of Fig. 3.The distributed load per unit length for the BWS method is $q=B I$ with $B$ the 


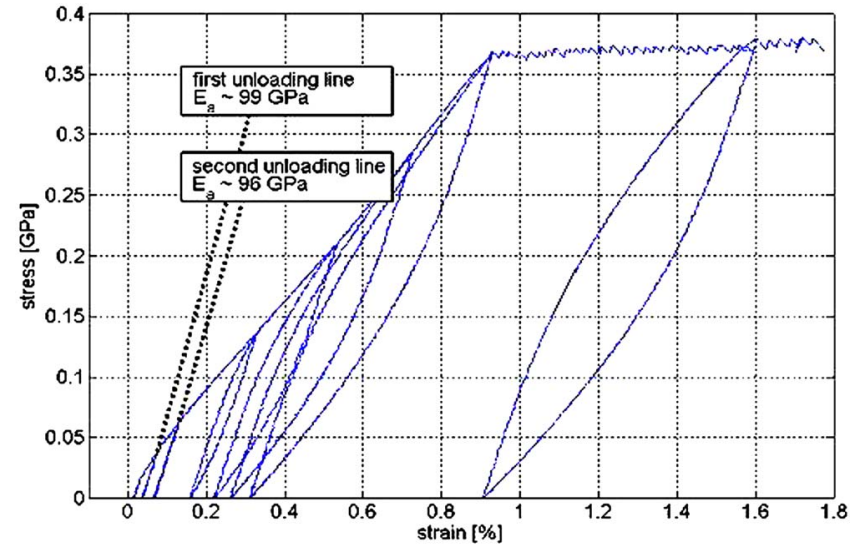

FIG. 4. Tensile stress-strain curve with unloading of a SMI-PIT sample measured with the TARSIS strand axial stress-strain probe at $4.2 \mathrm{~K}$.

applied magnet field and $I$ the strand current. The distributed load along the slot between the two changeovers to the supporting grooves in the barrel is then $Q$ (in Newton), with $Q=q L_{w}$.

The corresponding standard equation for the deflection $(f)$ in the case of distributed load $Q$, with clamped ends and length $L_{w}$ in Fig. 3, representing the BWS test condition is given by Eq. (5) with $\alpha=384$ and distributed load $Q$ instead of point load $F$. The deflection of the wire in the center of the slot is clearly less than for a point load due to the higher $\alpha$. For this distributed load, the value for $\beta$ is 24 in the center of the slot and 12 at the changeover from support to slot. ${ }^{30}$ As a result the peak bending strain at the changeover is higher than in the center of the slot. So for calculation of the peak bending strain we choose the lower value for $\beta$ and in order to compensate for nonideal clamping at the changeover, where the strand may be slightly lifted from the barrel, we choose $\beta=14$.

A complication is that the initial part of the axial tensile stress-strain curve of reacted $\mathrm{Nb}_{3}$ Sn strands, on which we determine the modulus of elasticity, hardly has a linear part or just up relatively low stress. ${ }^{24}$ The annealed copper (or bronze) in the wire yields from the beginning of the axial tensile stress-strain test and consequently it is essential to measure the axial stress-strain characteristic of the particular strand to enable an accurate computation of the mechanical response to applied bending loads. The stiffness of the strand, together with the sensitivity to strain, will actually determine the performance under bending load.

The axial tensile stress-strain curve is determined with the specially developed (TARSIS) probe ${ }^{24}$ on the SMI-PIT wire at $4.2 \mathrm{~K}$ and the result is plotted in Fig. 4. This characteristic is used to calculate the deflection of the wire in the bending tests for the given wavelengths.

As the linear region of the stress-strain curve for this wire is only linear for very low stress, the overall stressstrain behavior can only be described accurately by using elastoplastic properties. For simplicity in computation, but to model the strand axial elastoplastic behavior, the modulus $E$ is now approximated by two linear regimes. In the elastic

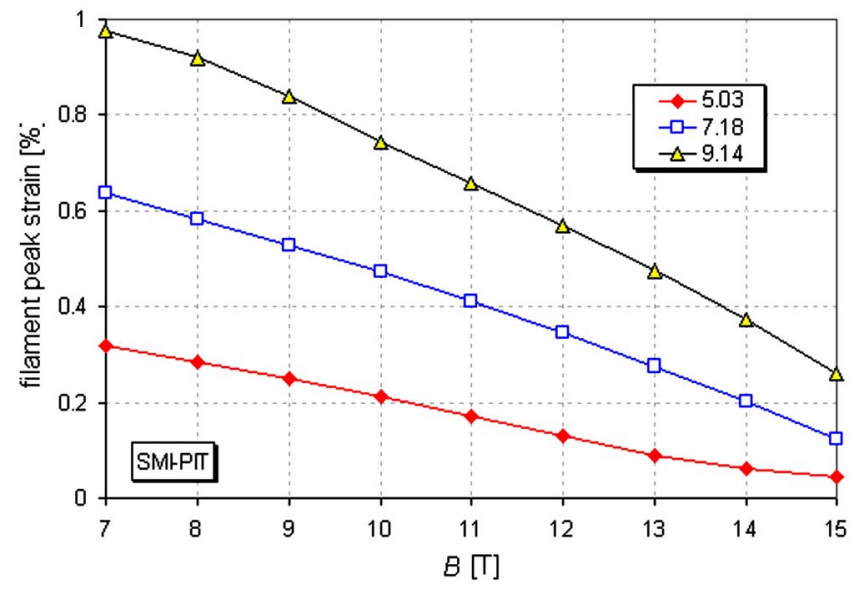

FIG. 5. The peak bending strain in the filamentary region of the strand at $I_{c}$ (10 $\mu \mathrm{V} / \mathrm{m})$ for three bending wavelengths (legend) vs the applied magnetic field.

region below an axial strain of $0.028 \%$ the modulus is taken by $100 \mathrm{GPa}$ while for $\varepsilon>0.028 \%$, with continuous yielding, the modulus is approached by $39 \mathrm{GPa}$.

An additional complication is the shift of the neutral axis but this is neglected here. These simplified elastoplastic model conditions were used previously to predict the loaddeflection curves for the TARSIS bending probe on the same strand type, showing good agreement with the experimental data. $^{23}$

For the BWS method, the peak bending strain is calculated for the applied magnetic fields and three bending wavelengths, taking into account the strand axial tensile stressstrain characteristics from Fig. 4 and relation (7) (see Fig. 5).

\section{EXPERIMENTAL RESULTS}

Figure 6 illustrates the effect of bending on the critical current $\left(I_{c, b}\right.$ bend section divided by $I_{c 0}$ supported section) for different $I_{c}$ criteria, 1,10 , and $100 \mu \mathrm{V} / \mathrm{m} . I_{c 0}$ is defined as the critical current without applied bending load while $I_{c, b}$ is the critical current with applied bending load. For Lorentz force directed inwards (regular current direction) we expect indeed a reduction in performance for lower field, i.e., higher

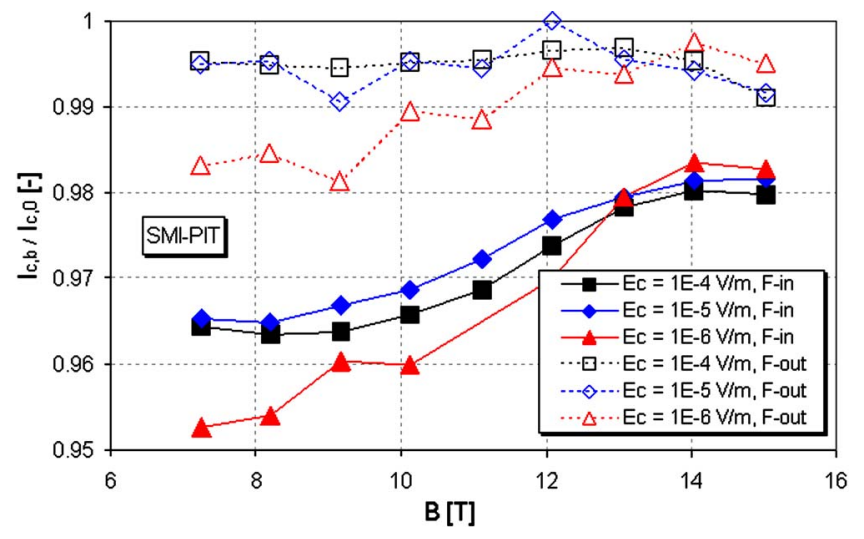

FIG. 6. The reduced $I_{c}$ for $E_{c}=1,10$, and $100 \mu \mathrm{V} / \mathrm{m}$ vs the applied field for regular current direction (F-in is force inwards) and reversed current direction (F-out) on turns with slots (bend) and fully supported; the wavelength is $5.0 \mathrm{~mm}$. 


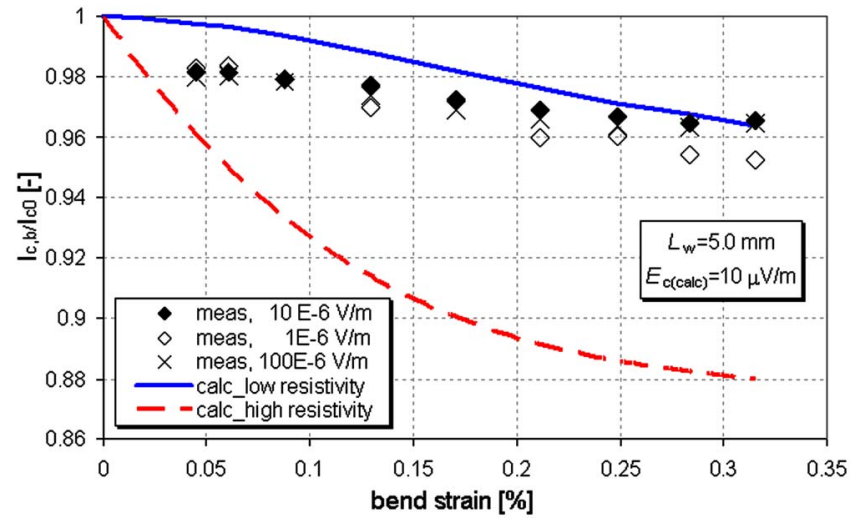

FIG. 7. The reduced $I_{c}$ vs the peak bend strain from turns crossing slots and entirely supported. The calculated reduced $I_{c}$ for low (lowRho) and high (highRho) transverse resistivities at $E_{c}=10 \mu \mathrm{V} / \mathrm{m}$ criterion are presented for comparison, the wavelength is $5.0 \mathrm{~mm}$.

bending force (see Fig. 5). The initial lowest electromagnetic load at $15 \mathrm{~T}$ already seems to lead to a reduction of $2 \%$.

A lower electric field criterion is linked to a higher sensitivity for bending. Initially, at low bending strain the $I_{c, b} / I_{c, 0}$ ratio is practically similar for 1,10 , and $100 \mu \mathrm{V} / \mathrm{m}$ while the difference in reduction becomes more pronounced with increasing force. When the current is reversed, starting again at highest applied field (lowest force), the $I_{c, b} / I_{c, 0}$ ratio is about 0.995 for both higher $E_{c}$ criteria while for $1 \mu \mathrm{V} / \mathrm{m}$ a small reduction gradually develops (the measurements were done after the ones with the force inwards).

In Fig. 7 the reduced $I_{c}$ is plotted versus the applied peak bending strain in the filamentary region for the three $E_{c}$ criteria. In the same graph we present the computed curves based on relations (1) and (2) for low and high strand transverse resistivities. The computation of these curves is based on the measured $I_{c}$ versus axial strain characteristic for an electric field criterion of $10 \mu \mathrm{V} / \mathrm{m}$ and the deviatoric strain scaling proposed by ten Haken. ${ }^{30}$

Although the tendency of the measured data seems not to intersect unity at zero bending strain, the overall performance corresponds clearly to the low transverse resistivity behavior.

The results for the barrel with $9.1 \mathrm{~mm}$ bending wave-

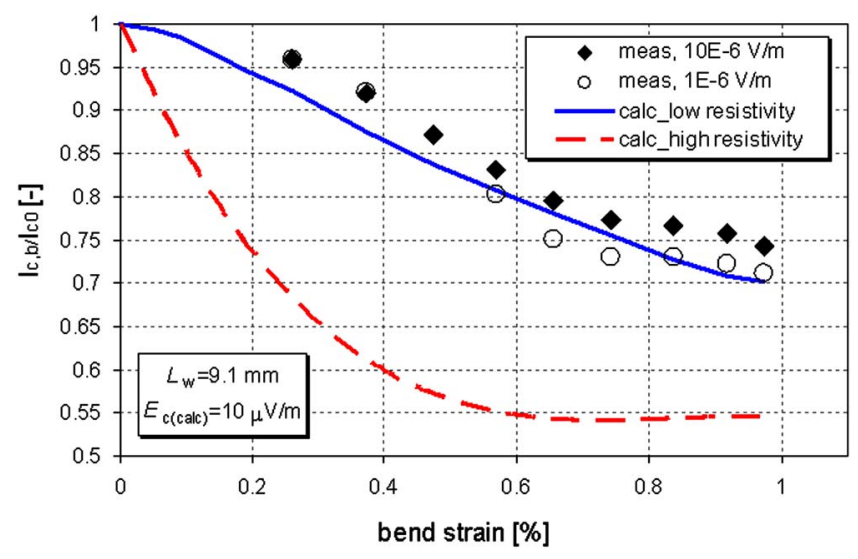

FIG. 8. The reduced $I_{c}$ vs the peak bend strain from turns crossing slots and supported. The calculated reduced $I_{c}$ for low and high transverse resistivities at $E_{c}=10 \mu \mathrm{V} / \mathrm{m}$ are presented for comparison, the wavelength is $9.1 \mathrm{~mm}$.

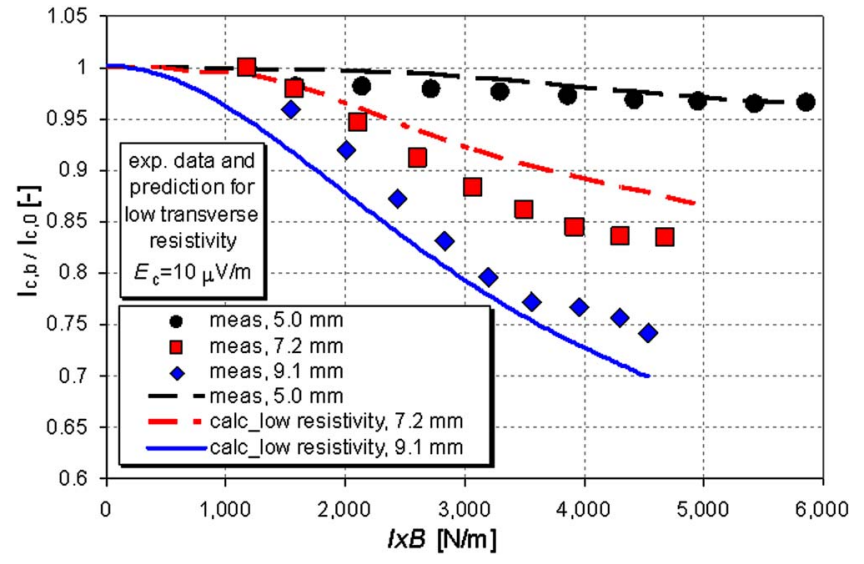

FIG. 9. The reduced $I_{c}$ vs the applied Lorentz force from turns crossing slots and entirely supported at wavelengths of $5.0,7.2$, and $9.1 \mathrm{~mm}$. The solid lines represent the predicted reduced $I_{c}$ for low transverse resistivity regime at $E_{c}=10 \mu \mathrm{V} / \mathrm{m}$.

length is presented in Fig. 8. A voltage level of $100 \mu \mathrm{V} / \mathrm{m}$ could not be achieved for the two largest wavelengths. Just as noticed for $5.0 \mathrm{~mm}$ wavelength, also for 7.2 and $9.1 \mathrm{~mm}$ wavelengths the measured reduced $I_{c}$ and the calculated curves, based on the bending strain relations for low and high strand transverse resistivity, show a response that matches the low transverse resistivity regime. Also here the tendency of the measured curves seems not to intersect unity at zero bending strain but in contradiction to the $5.0 \mathrm{~mm}$ wavelength, here an extrapolation would surpass unity.

The results for the three wavelengths are collected in Fig. 9 where the scale on the horizontal axis represents the $B I$ or Lorentz force with the unit $\mathrm{N} / \mathrm{m}$. In the same figure we plotted the computed curves based on relation (2) for low transverse resistivity and (7) to correlate with the bending strain.

\section{DISCUSSION}

The measured data, as summarized in Fig. 9, illustrate plainly a larger reduction in performance with longer bend-

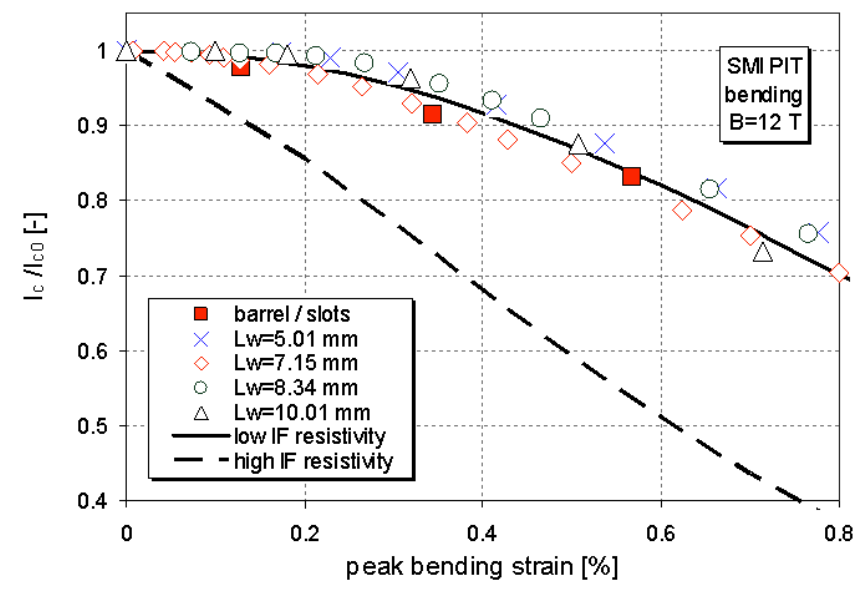

FIG. 10. The reduced $I_{c}$ vs the bending strain for the barrels with slots at wavelengths of 5.0, 7.2, and $9.1 \mathrm{~mm}$, compared with the data obtained on TARSIS bending probes with four wavelengths, all for $12 \mathrm{~T}$. The lines represent the calculated reduced $I_{c}$ for low (solid) and high (dashed) interfilament (IF) transverse resistivities. 


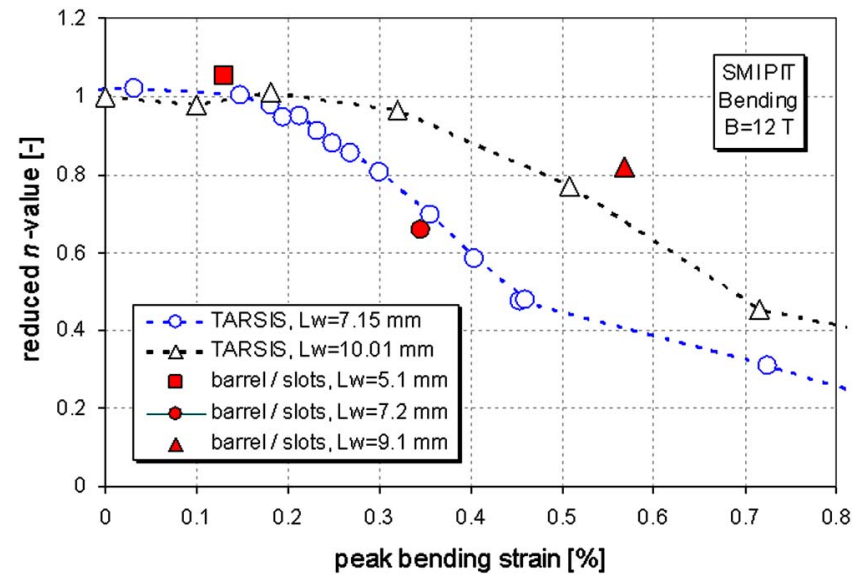

FIG. 11. The reduced $n$ value vs the bending strain on the BWS at wavelengths of 5.0, 7.2, and $9.1 \mathrm{~mm}$ compared with the results obtained with the TARSIS bending probes.

ing wavelength for increasing applied load or bending strain. Compared with the computations based on low transverse resistivity [relation (2)], the measured $I_{c}(\varepsilon)$ curve for axial strain, and the measured strand axial tensile stress-strain curve (from the TARSIS probe), represented by the solid lines in Figs. 7-9, we find a fair agreement between measured data and prediction. Altogether we find a difference of about 5\% with the largest deviation for the barrel with $9.1 \mathrm{~mm}$ wavelength. We observe that the data correspond with the prediction for low transverse resistivity, which in turn is in good agreement with the short current transfer length of $4 \mathrm{~mm}$ derived from the measured coupling-loss time constant.

This is also well illustrated in Fig. 10 where the data obtained at $12 \mathrm{~T}$ are collected from the advanced TARSIS bending probe ${ }^{23}$ and the BWS method. One of the main objectives of BWS test is to make a direct comparison to the results obtained with the TARSIS bending probes. The direct comparison shows excellent agreement between both experimental methods and with the computed curve for low transverse resistivity.

Although the number of data for the $n$ values determined at $12 \mathrm{~T}$ is only limited for the BWS method and a fair comparison is restricted, Fig. 11 confirms a rather good correlation for the $n$ value dependency against applied bending strain and wavelength. Both methods, BWS and TARSIS bending probe, show invariance or a slight enhancement of the $n$ value at low strain level and a higher $n$ value for the larger wavelength of $9.1 \mathrm{~mm}$ compared with $7.2 \mathrm{~mm}$.

\section{SUMMARY}

We developed and validated a simple and straightforward experimental method to verify the effect of bending due to electromagnetic load on the critical current of $\mathrm{Nb}_{3} \mathrm{Sn}$ strands. The method can be applied to strain sensitive conductors in general. The strand is wound on a standard ITER barrel with slots (BWS) to impose a spatial periodic bending pattern along the two center turns. The results of the method proposed here are, in terms of $I_{c}$ and $n$ value, in very good agreement with a more sophisticated method named TARSIS where beside transport properties, also the mechanical properties are monitored. The agreement between the measured and the predicted data for low interfilament transverse resistivity regime (short current transfer length) is about 5\%.

${ }^{1}$ J. W. Ekin, Cryogenics 20, 611 (1980).

${ }^{2}$ R. Zanino, N. Mitchell, and L. Savoldi-Richard, Cryogenics 43, 179 (2003).

${ }^{3}$ N. Mitchell, Cryogenics 43, 225 (2003).

${ }^{4}$ W. Specking and R. Flukiger, J. Phys. (Paris) 45, C1-79 (1984).

${ }^{5}$ C. R. Walters, L. M. Davidson, and G. E. Tuck, Cryogenics 26, 406 (1986)

${ }^{6}$ N. Cheggour and D. P. Hampshire, Rev. Sci. Instrum. 71, 4521 (2000).

${ }^{7}$ B. ten Haken, A. Godeke, and H. H. J. ten Kate, J. Appl. Phys. 85, 3247 (1999).

${ }^{8}$ A. Godeke et al., Rev. Sci. Instrum. 75, 5112 (2004).

${ }^{9}$ J. W. Ekin, J. Appl. Phys. 62, 4829 (1987).

${ }^{10}$ W. Specking, W. Goldacker, and R. Flukiger, Adv. Cryog. Eng. 34, 569 (1988).

${ }^{11}$ S. L. Bray and J. W. Ekin, Adv. Cryog. Eng. 38, 643 (1992).

${ }^{12}$ W. A. J. Wessel, A. Nijhuis, Y. Ilyin, W. Abbas, B. ten Haken, and H. H. J. ten Kate, International Cryogenic Materials Conference (ICMC), Anchorage, AL, 2004, edited by U. Balachandran (IOP, Melville, 2004), p. 466.

${ }^{13}$ Y. Nunoya, T. Isono, and K. Okuno, IEEE Trans. Appl. Supercond. 14, 1468 (2004).

${ }^{14}$ A. Nijhuis, Y. Ilyin, W. Abbas, B. ten Haken, and H. H. J. ten Kate, IEEE Trans. Appl. Supercond. 14, 1489 (2004).

${ }^{15}$ J. W. Ekin, Proceedings of Topical Conference on A15 Superconductors, 1980, (Plenum, New York, 1980), p. 187.

${ }^{16}$ B. J. Senkowicz, M. Takayasu, P. J. Lee, J. V. Minervini, and D. C. Larbalestier, IEEE Trans. Appl. Supercond. 15, 3470 (2005).

${ }^{17}$ L. Zani, H. Cloez, A. Della Corte, L. Muzzi, and A. Di Zenobio, Supercond. Sci. Technol. 18, S390 (2005).

${ }^{18}$ K. Itoh, T. Kuroda, and H. Wada, Physica C 382, 7 (2002).

${ }^{19}$ J. H. Schultz, L. Chiesa, D. L. Harris, P. J. Lee, J. V. Minervini, B. J. Senkowicz, M. Takayasu, and P. Titus, IEEE Trans. Appl. Supercond., 15, 1371 (2005).

${ }^{20}$ N. Mitchell, Cryogenics 42, 311 (2002).

${ }^{21}$ A. Nijhuis, Y. Ilyin, W. Abbas, B. ten Haken, and H. H. J. ten Kate, IEEE Trans. Appl. Supercond. 14, 1464 (2004).

${ }^{22}$ N. Mitchell, Physica C 401, 28 (2004).

${ }^{23}$ A. Nijhuis, N. C. van den Eijnden, Y. Ilyin, W. A. J. Wessel, A. den Ouden, and H. H. J. ten Kate, Supercond. Sci. Technol. 18, S273 (2005).

${ }^{24}$ N. C. van den Eijnden, A. Nijhuis, Y. Ilyin, W. A. J. Wessel, and H. H. J. ten Kate, Supercond. Sci. Technol. 18, 1523 (2005).

${ }^{25}$ S. Awaji, K. Watanabe, and K. Katagiri, Supercond. Sci. Technol. 16, 1059 (2003).

${ }^{26}$ P. Bruzzone, H. H. J. ten Kate, M. Nishi, A. Shikov, J. Minervini, and M. Takayasu, Advanced Cryogenic Engineering, Columbus OH, 1995, edited by L. T. Summers (Plenum, New York, 1996), p. 1351.

${ }^{27}$ J. W. Ekin, J. Appl. Phys. 49, 3406 (1978).

${ }^{28}$ G. Ries, IEEE Trans. Magn. 13, 524 (1977).

${ }^{29}$ A. M. Campbell, Cryogenics 22, 3 (1982).

${ }^{30}$ B. ten Haken, PhD Thesis, University of Twente, 1994. 